\title{
Géoarchéologie en contexte karstique dans la Maiella (Abruzzes, Italie)
}

Prospections à visée géoarchéologique et analyses non invasives de l'art rupestre

Elisa Nicoud, Guido Palmerini, Valentina Villa, Silvano Agostini, Lucile Beck, Rosa Maria Dessì, Xavier Gallet et Matthieu Lebon

\section{OpenEdition}

Édition électronique

URL : https://journals.openedition.org/baefe/4881

DOI : $10.4000 /$ baefe. 4881

ISSN : 2732-687X

Éditeur

ResEFE

\section{Référence électronique}

Elisa Nicoud, Guido Palmerini, Valentina Villa, Silvano Agostini, Lucile Beck, Rosa Maria Dessì, Xavier Gallet et Matthieu Lebon, « Géoarchéologie en contexte karstique dans la Maiella (Abruzzes, Italie) » [notice archéologique], Bulletin archéologique des Écoles françaises à l'étranger [En ligne], Italie, mis en ligne le 10 février 2022, consulté le 14 février 2022. URL : http://journals.openedition.org/baefe/4881 DOl : https://doi.org/10.4000/baefe.4881

Ce document a été généré automatiquement le 14 février 2022.

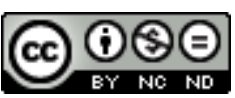

Le Bulletin archéologique des Écoles françaises à l'étranger est mise à disposition selon les termes de la Licence Creative Commons Attribution - Pas d'Utilisation Commerciale - Pas de Modification 4.0 International. 


\section{Géoarchéologie en contexte karstique dans la Maiella (Abruzzes, Italie)}

Prospections à visée géoarchéologique et analyses non invasives de l'art rupestre

Elisa Nicoud, Guido Palmerini, Valentina Villa, Silvano Agostini, Lucile Beck, Rosa Maria Dessì, Xavier Gallet et Matthieu Lebon

\section{NOTE DE L'AUTEUR}

Nous choisissons de garder la dénomination italienne des communes et des sites, incluant « grottes, abri, carrière ou paroi » (« Grotta, Riparo, Cava, Parete »).

Date précise de l'opération : 23 août-12 septembre 2021

Numéro de mission : $1^{\mathrm{e}}$ mission

Composition de l'équipe de terrain : Elisa Nicoud, Valentina Villa, Guido Palmerini (CNRS, Université Côte d'Azur, UMR 7264 CEPAM), Lucile Beck (LMC14, LSCE/IPSL, CEA-CNRS-UVSQ, Université Paris-Saclay), Xavier Gallet, Matthieu Lebon (MNHN, Musée de l'Homme, UMR 7194 HNHP), Mariano Antonio Spera, Dino D'Alessandro (Parco nazionale della Maiella), Fabio Fusco (Palynologue indépendant), Massimo Mariani et Claudio Pagone (Soccorso alpino e Speleologico Abruzzo, CNSAS), Deneb Teresa Cesana (Soprintendenza Archeologia Belle Arti e Paesaggio per le Province di Chieti e Pescara), Rita Silvaggi, Valerio Natarelli.

Partenariats institutionnels : École française de Rome, CNRS CEPAM UMR 7264, Université Côte d'Azur Idex ${ }^{\text {Jedi }}$, Parco nazionale della Maiella, Soprintendenza Archeologia Belle Arti e Paesaggio per le Province di Chieti e Pescara.

Établissement éditeur : EFR

Établissements porteurs de l'opération : EFR 
Nos remerciements les plus sincères sont adressés à Lucio Zazzara, Luciano Di Martino (Parco Nazionale della Maiella), Silvano Agostini (Parco Nazionale della Maiella), Rosanna Tuteri, Deneb Cesana (Soprintendenza Archeologia, Belle Arti e Paesaggio per le Province di Chieti e Pescara). Nous remercions Alfonso Palmerini et Luciana Confalone pour leur accueil ainsi que Claudio Pagone et Massimo Mariani (Soccorso alpino e Speleologico Abruzzo, CNSAS) pour avoir assuré notre sécurité et à Tommaso Mattioli pour ses conseils précieux.

Les recherches 2021 dans les grottes et abris sous roches de la Maiella sont financées par l'École française de Rome, le laboratoire CNRS CEPAM UMR 7264, l'Université Côte d'Azur Idex ${ }^{\text {Jedi, avec }}$ le soutien du Parco nazionale della Maiella.

\section{Extension des recherches archéologiques de Valle Giumentina à l'ensemble de la Maiella}

1 Le programme de recherche mené à Valle Giumentina ${ }^{1}$ depuis 2012 a ouvert la voie à de nouvelles et fructueuses enquêtes archéologiques dans le Parc national de la Maiella (PNM), dont l'ampleur et la réalisation concrète n'auraient pu être anticipées au début du projet sur ce gisement préhistorique. En 2021, le PNM a obtenu le label Geopark Unesco ${ }^{2}$, mettant ainsi en valeur son riche patrimoine géologique. Valle Giumentina prend ainsi place parmi les sites d'intérêt majeur du Parc, en raison de son enregistrement sédimentaire couvrant plusieurs cycles glaciaire-interglaciaire du Pléistocène moyen ${ }^{3}$, et conservant de nombreux vestiges d'occupations humaines $\mathrm{du}$ Paléolithique inférieur. L'opportunité d'élargir nos recherches sur la Préhistoire de la Maiella nous est offerte par l'émergence de différents projets portant sur le réseau karstique de ce massif calcaire. D'une part, le projet Interreg intitulé "adriaticaves » vient de s'achever ${ }^{4}$. Il était porté par le PNM et plusieurs institutions internationales de la région adriatique et ionienne. Il a livré une meilleure connaissance de la géologie et de la topographie des grottes, utile pour y mener de nouveaux travaux archéologiques. D'autre part, l'étude de l'art rupestre du massif fait l'objet d'un projet post-doctoral ${ }^{5}$ depuis 2020. Il s'agit de représentations peintes ou gravées s'échelonnant de la Préhistoire au Moyen Âge.

2 Forts de cette dynamique et de l'important soutien du PNM pour les recherches archéologiques, nous avons pu développer un programme de prospection dans les grottes et abris du massif, à la recherche d'indices du Paléolithique et de séquences sédimentaires du Pléistocène, susceptibles de compléter en diachronie les données obtenues à Valle Giumentina. Bien que disposant d'archives géoarchéologiques couvrant une durée de 150000 ans (MIS 16 à 12), ce site exceptionnel ne constitue qu'un morceau (crucial) du puzzle de l'histoire environnementale et culturelle de l'Italie centrale et de l'Europe au Pléistocène. Cette extension géographique et géomorphologique de nos recherches dans les Abruzzes est également l'un des objectifs du programme quinquennal de l'EFR 2022-2026 sur le Paléolithique d'Italie (axe 1, l'Italie centrale). Nous appuyant sur les travaux antérieurs des archéologues et des géologues, la première mission centrée sur les grottes a pris place du 23 août au 12 septembre 2021. Afin de mutualiser les efforts logistiques et de profiter des guides et connaisseurs du terrain, nous avons ainsi mené de front des reconnaissances géoarchéologiques et des analyses non invasives des peintures rupestres dont la méthodologie et les premiers résultats sont présentés ci-après. La rareté des études archéologiques dans la Maiella depuis les années 1970 en fait un territoire encore riche 
de vestiges méconnus, voire inconnus. Notre opération veut non seulement réviser des travaux anciens mais aussi livrer un pan de connaissance totalement inédit des occupations humaines du passé dans cette zone. Il nous revient en premier lieu de déterminer leur nature et leur âge.

\section{Remarques sur le karst de la Maiella}

3 Le massif montagneux de la Maiella se trouve au centre de la chaîne apennine et se développe en parallèle de la côte adriatique, sur près de $35 \mathrm{~km}$ de long selon un axe $\mathrm{N} /$ S. Constitué de vallées, plateaux et hauts sommets, son point culminant (Monte Amaro, $2793 \mathrm{~m}$ ), est le deuxième plus haut sommet de tous les Apennins ${ }^{6}$. La Maiella est constituée de sédiments carbonatés déposés à partir du Cénozoïque ${ }^{7}$. La karstification y est récente, du fait de l'orogénie plio-quaternaire du massif. Les formes les plus anciennes se trouvent au sud du massif. Différents épisodes de soulèvement du massif, en particulier durant le Pléistocène moyen, ont engendré un karst étagé et ont forgé les canyons actuels. Ceux-ci sont alimentés abondamment par les eaux de fonte des neiges. Ces canyons peuvent être très profonds (env. 150 m), comme ceux de Santo Spirito/San Bartolomeo et de l'Orta ${ }^{8}$ (fig. 1). Ils sont exploités par les populations humaines pour l'eau, le silex, le bitume ou le calcaire, y compris à l'époque contemporaine. Le long de ces canyons, on trouve un système de grottes et d'abris naturels superposés en trois étages. Les grottes sont maintenant toutes inactives mais témoignent de l'activité du réseau karstique avant l'ouverture des canyons. Par ailleurs, en sus d'importants mouvements tectoniques, les phénomènes de dissolution karstique ont favorisé la création de cirques et de vallées glaciaires en altitude.

Dans la zone concernée par le projet, correspondant au territoire de l'intégralité des communes membres du PNM et de quelques communes limitrophes, 110 grottes naturelles sont recensées auxquelles il faut ajouter des abris innombrables, plus ou moins profonds.

\section{Les recherches archéologiques en grottes et abris dans la Maiella}

\section{Les sites archéologiques en grottes et abris}

5 Grottes et abris présentent un intérêt non seulement géologique mais aussi archéologique (Fig. 1 et 2). Des occupations humaines y sont identifiées depuis le Paléolithique. 34 sites ont fait l'objet de fouilles archéologiques, 28 en grottes et 6 en contexte d'abris. Il faut ajouter 30 sites d'art rupestre en grottes et abris, principalement constitués de peintures à pigments rouge ou noirs, déjà recensés dans le massif et ses environs immédiats. Leur âge reste à préciser mais ils devraient s'étendre de la fin du Paléolithique au Moyen Âge. La Maiella compte ainsi la plus haute concentration de sites d'art rupestre de l'Italie apennine'

\section{Les sites du Paléolithique et du Néolithique}

C'est à A. M. Radmilli, l'archéologue des premières fouilles à Valle Giumentina dans les années 1950, que l'on doit la plupart des fouilles en grotte dans la Maiella, tant sur des 
sites du Paléolithique que du Néolithique ${ }^{10}$. Ses élèves, devenus chercheurs de l'Université de Pise, ont ensuite poursuivi les travaux dans la région, en particulier Carlo Tozzi et Giovanni Boschian ${ }^{11}$. Les derniers travaux en grotte ont pris place au début des années $2000^{12}$.

7 L'occupation des grottes ne semble débuter qu'au Paléolithique moyen. Des vestiges lithiques de conception Levallois et des restes fauniques associés sont apparus notamment à Grotta dei Mandroni ${ }^{13}$ à Serramonacesca, dans le Vallone del Foro ${ }^{14}$ à Pretoro, dans la Grotta degli Orsi Volanti ${ }^{15}$ à Rapino, où les restes d'un macaque ont été découverts associés à des restes fauniques plus habituels pour la fin du Pléistocène supérieur. Cette occupation tardive des grottes peut résulter de l'histoire géologique du massif et de l'ouverture tardive du réseau karstique et des canyons accessibles actuellement.

8 Le Paléolithique supérieur est présent également au nord-est du massif (fig. 1). La Grotta Eremo Sant'Angelo ${ }^{16}$ à Lettomanopello est le seul témoignage de Gravettien dans la région, alors que Cava Pretoro ${ }^{17}$ aussi nommée Grotta del Foro 2 à Pretoro, Grotta Rutina $^{18}$ à Guardiagrele, Grotta del Colle ${ }^{19}$ à Rapino et Riparo San Bartolomeo ${ }^{20}$ à Roccamorice sont attribuées à l'Épigravettien.

La Grotta dei Piccioni ${ }^{21}$ à Bolognano est l'un des sites majeurs du Néolithique d'Italie centrale. À proximité, la Grotta Scura ${ }^{22}$ à Bolognano a livré également des céramiques néolithiques et postérieures. Des vestiges du Néolithique ont été fouillés également à Grotta del Colle ${ }^{23}$ à Rapino.

L'occupation humaine des milieux karstiques se poursuit selon d'autres modalités à l'Âge des métaux puis jusqu'au Moyen Âge. Durant cette dernière période, la Maiella et la montagne voisine du Morrone accueillent des ermites, en particulier la figure de Pietro da Morrone, devenu Pape en 1294 sous le nom de Célestin V $\mathrm{V}^{24}$. Sous la direction de l'Abbaye de San Clemente a Casauria, des villages fortifiés sont bâtis dans la Maiella qui devient le centre de l'art roman des Abruzzes ${ }^{25}$. Une nouvelle économie basée sur l'élevage ovin et la production de laine se met en place. À l'époque moderne jusqu'à très récemment, certains abris restent occupés par les troupeaux et leurs bergers.

11 Les reconnaissances géoarchéologiques menées durant la mission intéressent uniquement la Préhistoire. Toutes les grottes de la Maiella n'ont pas été explorées. Celles que nous avons citées comportent toutes une séquence stratigraphique plurimétrique. D'autres grottes seraient donc susceptibles de contenir des vestiges archéologiques du Paléolithique. Étudiées anciennement pour la plupart, ces séquences stratigraphiques méritent un réexamen chronostratigraphique et paléoenvironnemental pour mieux restituer les interactions des Hommes avec ce milieu montagnard durant le Pléistocène. 
Fig. 1. Localisation des sites archéologiques en grottes et abris de la Maiella.

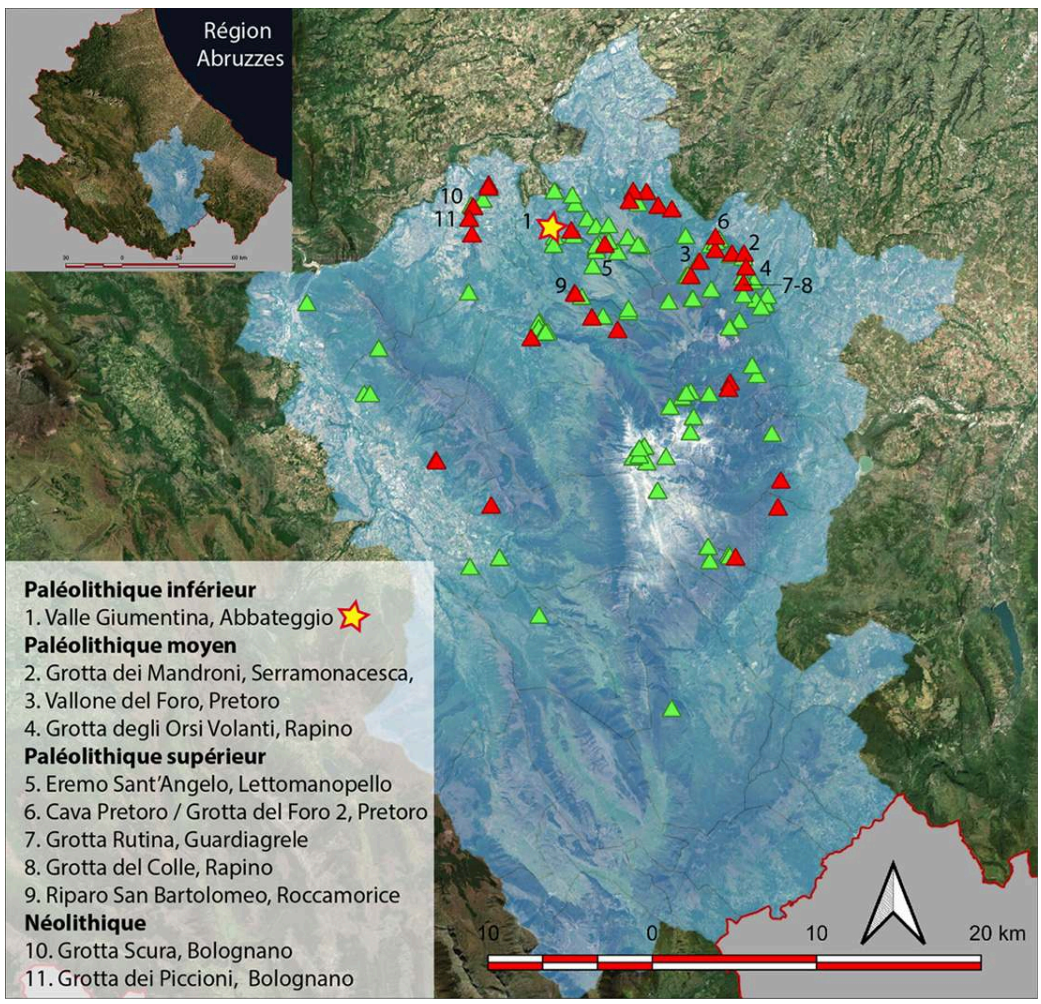

La zone en bleu correspond aux communes du Parc national de la Maiella. Les triangles rouges indiquent les grottes et abris à indices d'occupations archéologiques et les triangles verts, les grottes qui en sont dépourvues dans l'état actuel de nos connaissances. Les sites numérotés correspondent à Valle Giumentina ( $\left.n^{\circ} 1\right)$ ainsi qu'aux sites du Paléolithique et du Néolithique en domaine karstique ayant fait l'objet de fouilles.

SIG, G. Palmerini/PNM et DAO, E. Nicoud/EFR/CEPAM, d'après une cartographie inédite de S. Agostini.

\section{Les sites d'art rupestre}

12 Un autre aspect de la recherche en contexte karstique regarde l'art rupestre. L'état des connaissances est assez maigre; pourtant la Maiella comporte la plus grande concentration de peintures de toute la chaîne apennine, puisque 30 sites ont déjà été identifiés. L'art rupestre post-glaciaire en Italie comprend les célèbres peintures de Porto Badisco $^{26}$ dans le canal d'Otranto ou encore les gravures alpines du Valcamonica ${ }^{27}$. L'art rupestre des Apennins est moins connu. En Italie centrale, les premières études consacrées à ces représentations ont débuté dans les années 1960 avec la découverte de l'abri de Pacentro $^{28}$ (fig. 2 et 7). Le travail s'est poursuivi avec l'engagement de l'Université de Pise qui définit en 2015 les caractéristiques générales du phénomène ${ }^{29}$. Il s'agit notamment de figures schématiques et de symboles abstraits gravés ou peints en rouge ou en noir, qui sont systématiquement réalisés dans de petits abris, sur des falaises ou des rochers avec vue panoramique, offrant un contrôle des cols et de vastes étendues. Les études les plus récentes ${ }^{30}$ indiquent que les peintures et gravures de cette région ont des affinités stylistiques étroites avec l'art schématique de la France méditerranéenne et du Levant espagnol datant de la Protohistoire ${ }^{31}$. Toutefois, des figures de cavaliers indiquent également la période médiévale (Riparo di Santo Spirito ${ }^{32}$ ). Aucune datation directe n'a été effectuée jusqu'à présent et, faute de prospections systématiques, nous ne connaissons à ce jour qu'une partie des peintures 
et gravures de la Maiella. Certaines ont été signalées aux autorités mais n'ont pas été inventoriées.

Fig. 2. Localisation des sites d'art rupestre en grottes et abris de la Maiella.

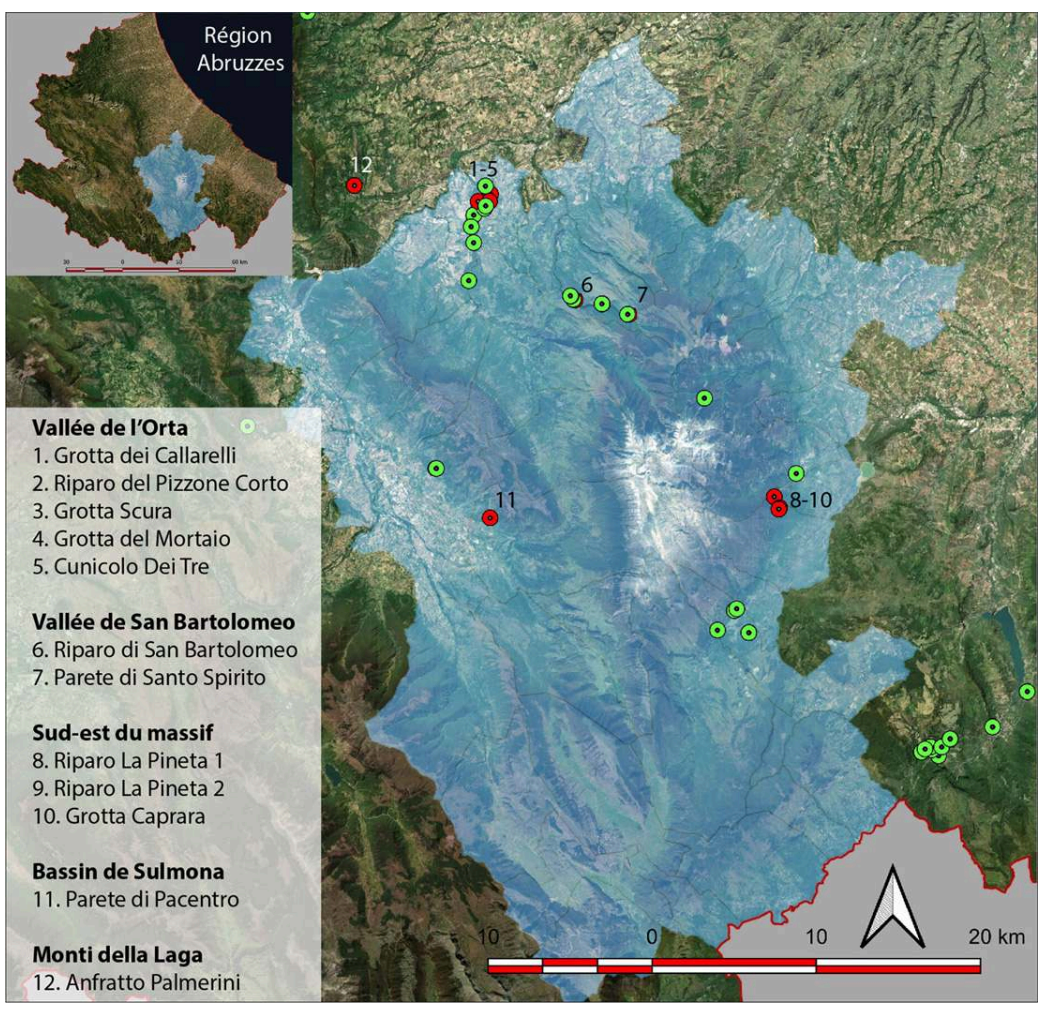

La zone en bleu correspond aux communes du Parc national de la Maiella. Les ronds rouges indiquent les grottes et abris à peintures rupestres explorés lors de la mission 2021 et les ronds verts, les autres sites connus d'art rupestre.

SIG, G. Palmerini/PNM et DAO, E. Nicoud/EFR/CEPAM, d'après une cartographie inédite de S. Agostini.

\section{La mission 2021 sur le terrain}

\section{Déroulement}

Douze grottes, abris et parois ont été explorés durant la mission (fig. 2). Ces sites d'art rupestre sont situés essentiellement sur le versant nord-ouest de la Maiella (Grotta dei Callarelli, Riparo del Pizzone Corto, Grotta Scura, Grotta del Mortaio, Riparo di San Bartolomeo, Parete di Santo Spirito, Cunicolo Dei Tre) et plus rarement sur les versants sud-est (Riparo La Pineta 1, Riparo La Pineta 2, Grotta Caprara) et ouest sur le Monte Morrone (Riparo di Pacentro). Une anfractuosité a fait également l'objet de relevés au nord du fleuve Pescara (Anfratto Palmerini). L'objectif de la mission était donc double, rechercher des séquences sédimentaires susceptibles de conserver des dépôts du Pléistocène et des vestiges du Paléolithique, et analyser et éventuellement échantillonner les pigments utilisés pour la réalisation des peintures rupestres. 


\section{Les dépôts sédimentaires observés}

14 l'oligocène et du Miocène supérieur, plusieurs grottes présentent un intérêt géologique et préhistorique. Certaines conservent les traces d'anciens conduits karstiques, qui fonctionnaient avant l'ouverture du canyon ${ }^{33}$. Par exemple, au fond de la Grotta Scura, un conglomérat calcaire est composé d'éléments typiques de la série du Monte Morrone, pourtant aujourd'hui déconnecté de la Maiella par la vallée de l'Orta. Les galets sont roulés et leurs dimensions et caractéristiques pétrographiques rappellent ceux des terrasses de $1^{\text {er }}$ et $2^{\text {ème }}$ ordres du Val Pescara. La Grotta Scura est une ample cavité longue de $350 \mathrm{~m}$, qui présente au moins trois phases de creusement en pleine charge hydrique, entrecoupées de phases de remplissage sédimentaire partiel. Certains de ces dépôts sont conservés, concrétionnés et couverts d'une patine de fer et manganèse (fig. 3A). Des prélèvements sont envisagés pour mieux cerner leur chronologie.

Toujours sur la rive droite de l'Orta, la Grotta dei Callarelli, profonde de $50 \mathrm{~m}$, est célèbre pour l'empreinte de main positive droite peinte en noir sur une paroi ${ }^{34}$ (fig. 3B). C'est une grotte difficile d'accès découverte par plusieurs explorateurs dans les années $1960^{35}$. Un sondage a été effectué en 1965. Le remplissage sédimentaire de la grotte ne semble pas comprendre de vestiges archéologiques. Il se compose de dépôts clastiques remobilisés dans une matrice argilo-limoneuse. La grotte semble avoir été une résurgence active jusqu'à très récemment ${ }^{36}$. Des peintures noires sont distribuées le long de toutes les parois et sont majoritairement recouvertes d'incrustations carbonatées, qui souvent les rendent peu visibles. Des analyses chimiques ont été conduites par Ezio Burri ${ }^{37}$ en 1977 sur des peintures de la grotte voisine du Mortaio et dans la Grotta dei Callarelli. Toutefois nous n'avons pas retrouvé les peintures en question dans cette dernière grotte, hormis l'empreinte de main. Les analyses de cette peinture en 1977 ont montré la présence de strontium. Cet élément n'a pas été identifié lors de nos mesures pXRF dans la Grotta dei Callarelli, mais il est présent sur d'autres panneaux de la région, associés au calcium et au souffre indiquant la présence de gypse (formule chimique $\mathrm{CaSO}_{4} \cdot 2 \mathrm{H}_{2} \mathrm{O}$ ). Sur la main, le calcium est majoritaire et un pic de soufre apparait dans le spectre. 
Fig. 3. Vues de grottes de la Maiella explorées durant la mission 2021.
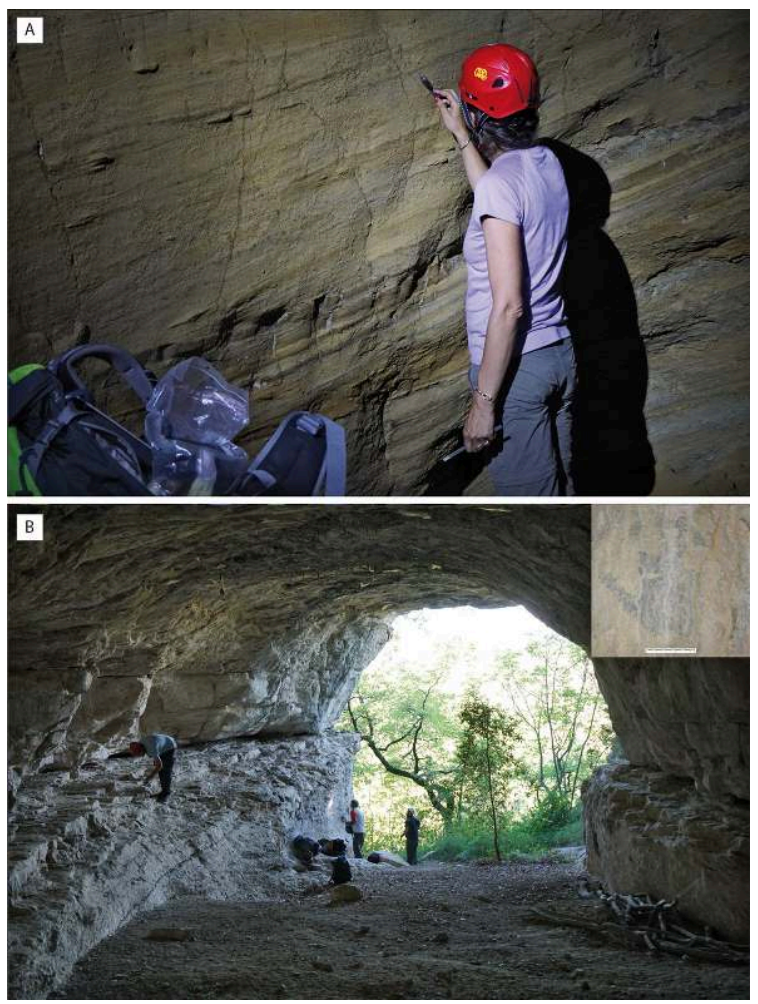

A. Vue de sédiments alluviaux pléistocènes stratifiés préservés dans la Grotta Scura à Bolognano

B. Vue depuis l'intérieur de Grotta dei Callarelli et vue de détail de la main peinte. La surface topographique actuelle est constituée de dépôts clastiques et de ruissellement. Les découvreurs ont réalisé un sondage dans la grotte en 1965.

E. Nicoud/EFR/CEPAM.

\section{Les analyses non invasives de l'art rupestre}

Une première étape du travail consiste en des analyses réalisables in situ, non invasives. Les analyses élémentaires ont été réalisées grâce au spectromètre de fluorescence des rayons X portable Elio (pXRF; XGLAB-Bruker) du plateau de Caractérisation des Archéomatériaux et des Archives Sédimentaires (C2AS) de l'UMR Histoire naturelle de l'Homme préhistorique. Ce système est composé d'un générateur de rayon $\mathrm{X}$ constitué d'une anode Rh dont le rayon est collimaté sur l'échantillon sur une zone de $1.2 \mathrm{~mm}$ de diamètre. La distance de travail de $1.4 \mathrm{~cm}$ permet une analyse sans contact. Un détecteur SDD (Silicon Drift Detector) de $25 \mathrm{~mm}^{2}$ permet l'enregistrement des énergies de 1 à $30 \mathrm{keV}$ avec une résolution de l'ordre de $140 \mathrm{eV}$. Pour chaque figure, une à trois zones ont été analysées afin de prendre en compte l'hétérogénéité des matériaux. Des analyses ont également été réalisées sur le calcaire de la paroi et dans des zones d'altération pour comparaison. Le logiciel $\mathrm{PyMca}^{38}$ est utilisé afin d'effectuer le traitement des spectres acquis et évaluer les concentrations des différents éléments identifiés qui seront présentées dans un rapport ultérieur. 
Fig. 4. L'instrument pXRF Elio (XGLab - Bruker) utilisé pour l'analyse des pigments.
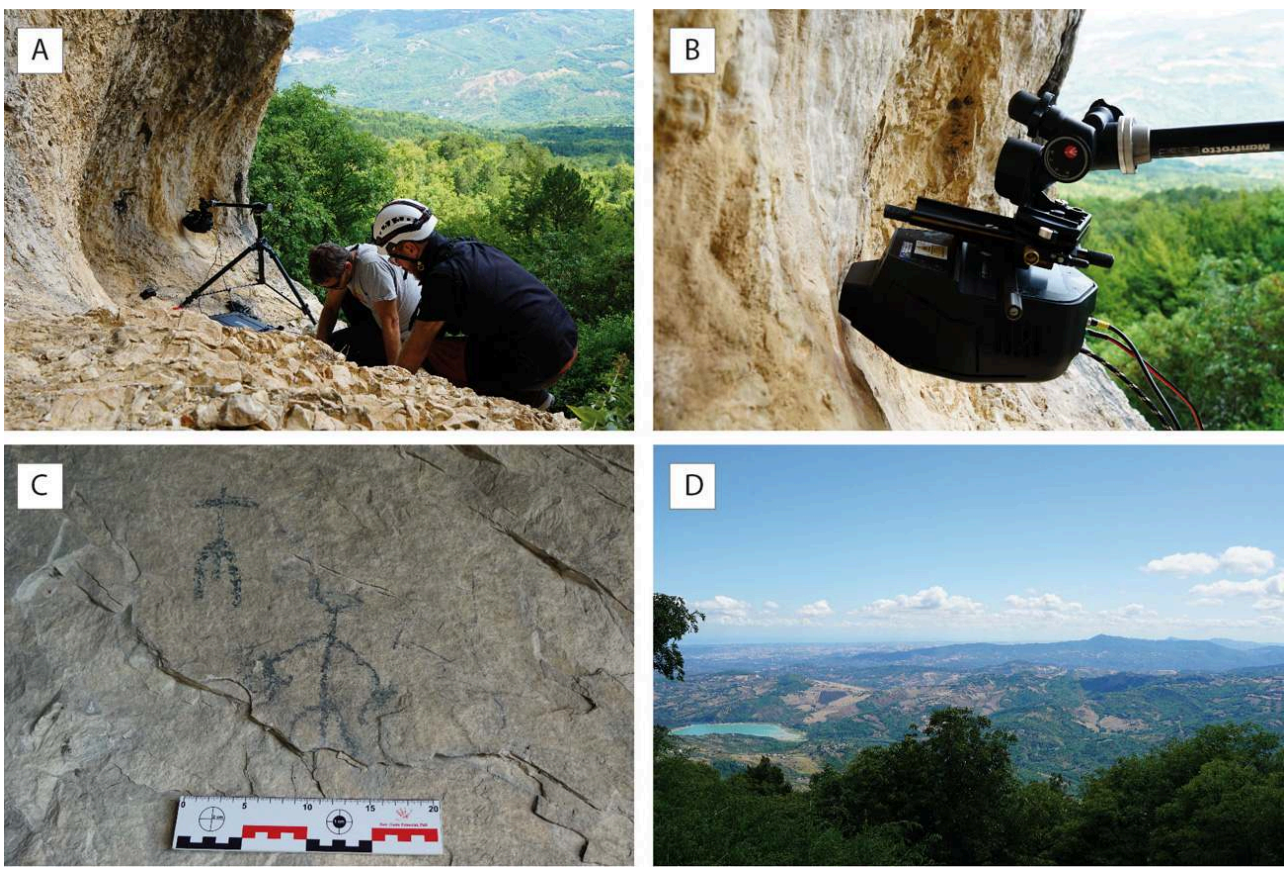

A. Vue de l'utilisation du pXRF relié à un ordinateur à Grotta Caprara à Lama dei Peligni. B. Vue de détail de l'appareil qui doit être positionné à distance minimale de la paroi pour réaliser les analyses élémentaires des pigments ou des coulées de calcite in situ et sans contact avec la peinture.

C. Peintures de la grotte. D. Panorama très ouvert depuis la grotte.

E. Nicoud/EFR/CEPAM.

Grâce à la spectrométrie par fluorescence à rayons X, il a été possible de reconnaître dans 12 sites, la présence d'oxalates de calcium, riches en carbonates, qui se forment par l'action de micro-organismes sur les parois. La datation de ces composants organiques pourrait ainsi fournir un âge minimal des peintures recouvertes de concrétions calcaires. Cette approche a déjà été appliquée avec succès sur des sites de l'Holocène en Australie et en Espagne. Des tests effectués en Namibie par le laboratoire LMC14 ont démontré la faisabilité des datations de ces couches d'altération à partir d'échantillons de $400 \mathrm{mg}^{39}$. Par ailleurs, plusieurs peintures au charbon ont été identifiées en vue de prélèvements pour des datations directes. 
Fig. 5. Exemples de grottes ornées de la Maiella.
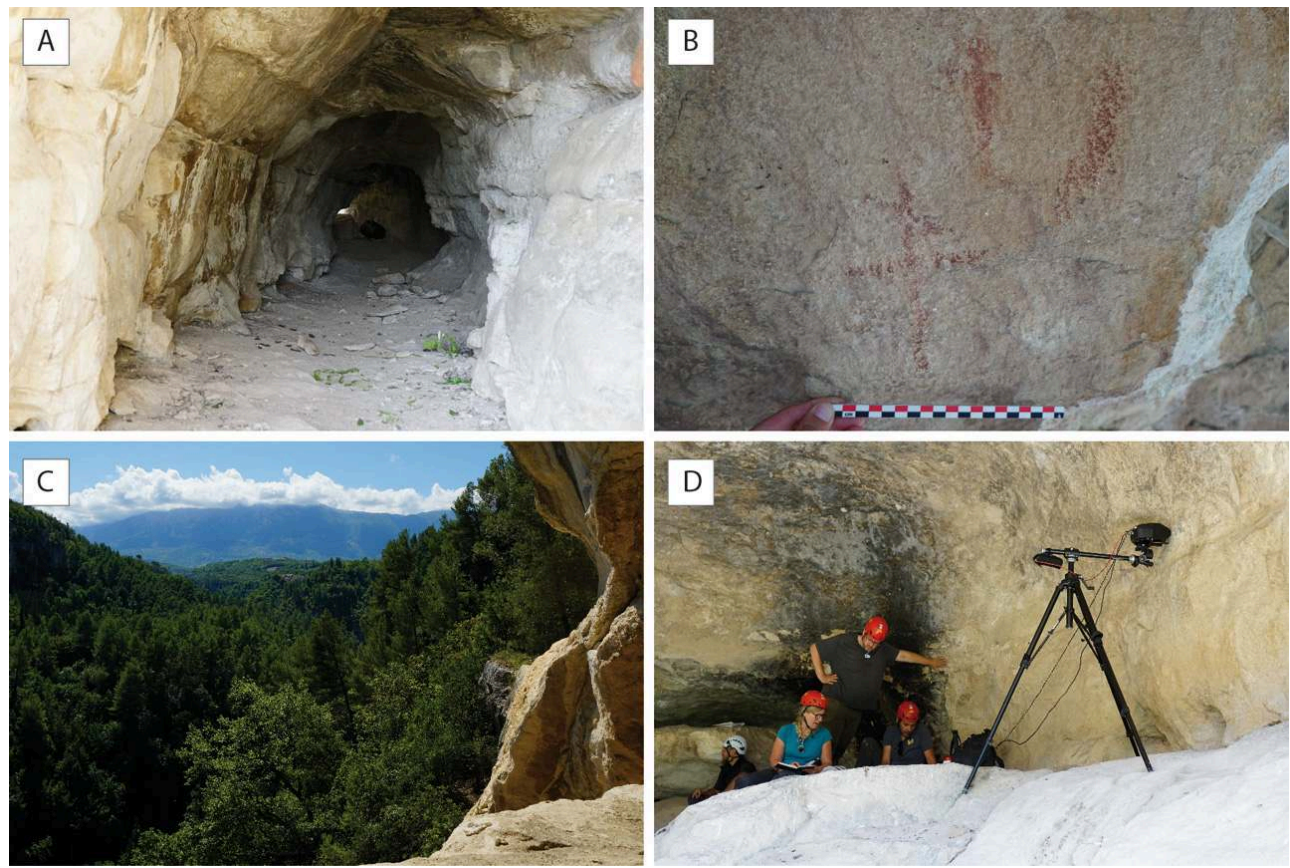

A. Vue du Cunicolo dei Tre. B. Motifs cruciformes. C. Canyon boisé de l'Orta, profond de $150 \mathrm{~m}$ environ. D. Analyses en cours à Grotta del Mortaio à Bolognano.

E. Nicoud/EFR/CEPAM.

Les analyses pXRF ont permis d'obtenir de premières informations sur la nature et la composition des matières colorantes employées pour la réalisation des peintures. Les pigments rouges sont à base d'oxydes de fer, de l'hématite associée en proportion plus ou moins importantes à des aluminosilicates. Nous avons pu distinguer trois types de pigments rouges. Le premier pigment correspond à un oxyde de fer relativement pur, contenant peu d'aluminosilicates et peu d'éléments mineurs et traces. Le second a une composition proche du premier, avec peu d'aluminosilicates, mais il contient en revanche des traces d'arsenic (fig. 6A-C). Le troisième correspond à un pigment rouge orangé, contenant une part plus importante d'aluminusilicates, ainsi que des éléments traces tels que le titane, le manganèse et le chrome. L'analyse de peintures de balisage des sentiers de randonnées du Parc national livre un spectre bien différent, avec notamment la présence de plomb dans la peinture (fig. 6D).

Les pigments noirs sont constitués exclusivement de carbone, probablement du charbon. Les couches de pigment sont relativement épaisses pour certaines figures et la matière colorée pourrait être prélevée afin de faire l'objet d'une datation radiocarbone. Nous avons d'ailleurs pu observer sur plusieurs sites que les panneaux étaient affectés par importantes desquamations qui entraîneront à terme la disparition complète des peintures. C'est principalement le cas sur les panneaux les plus exposés aux intempéries. Des prélèvements pourraient donc s'intégrer dans une démarche conservatoire en association avec des relevés photographiques. 
Fig. 6. Exemple d'analyses pXRF sur les peintures de la vallée de l'Orta.

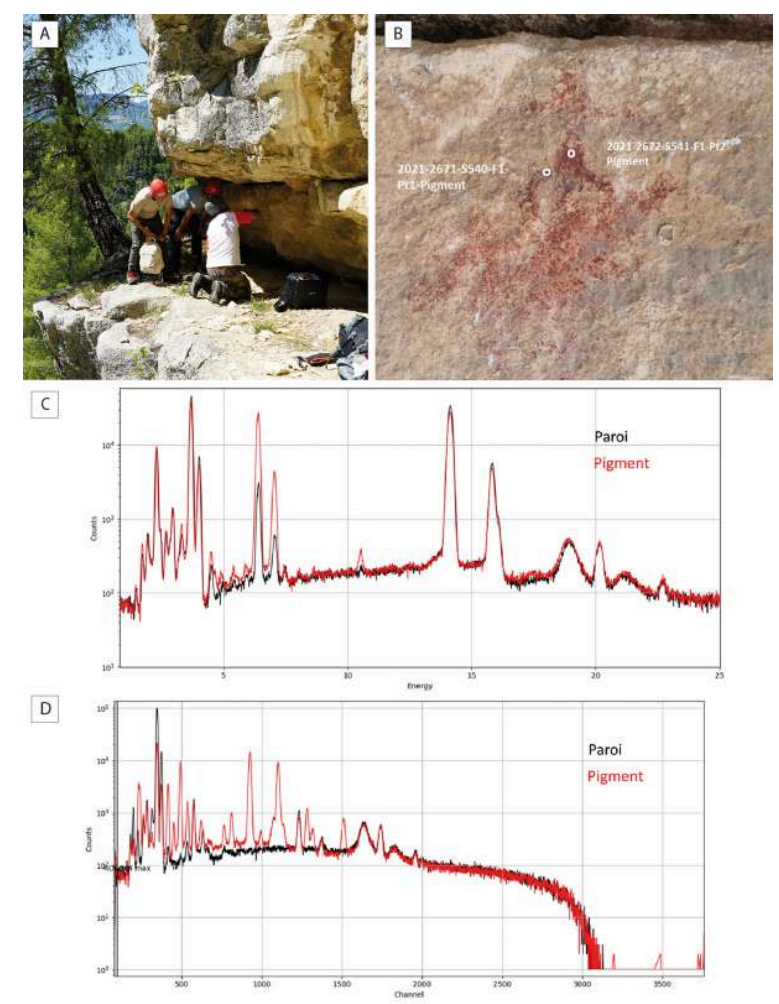

A. Abri du Pizzone Corto. B. Photographie de la peinture et localisation des analyses XRF.

C. Comparaison des spectres XRF du pigment de la figure rouge riche en fer du site avec celui de la paroi calcaire. D. Spectre XRF de pigment de peinture moderne riche en plomb, utilisée pour signaler les sentiers de randonnée.

A. E. Nicoud/EFR/CEPAM ; B-D. M. Lebon, X. Gallet/PNM/MNHN.

Par ailleurs, nous réalisons des relevés 3D des sites et des panneaux de peintures à haute résolution, à l'aide d'un appareil photographique, d'un drone équipé d'un GPS et d'un distancemètre (Leica Disto D810 Touch à dispositif FTA 630). Les images sont traitées avec les logiciels Agisoft Metashape Professional et Autocad (fig. 7). 
Fig. 7. Relevé des peintures de Pacentro.
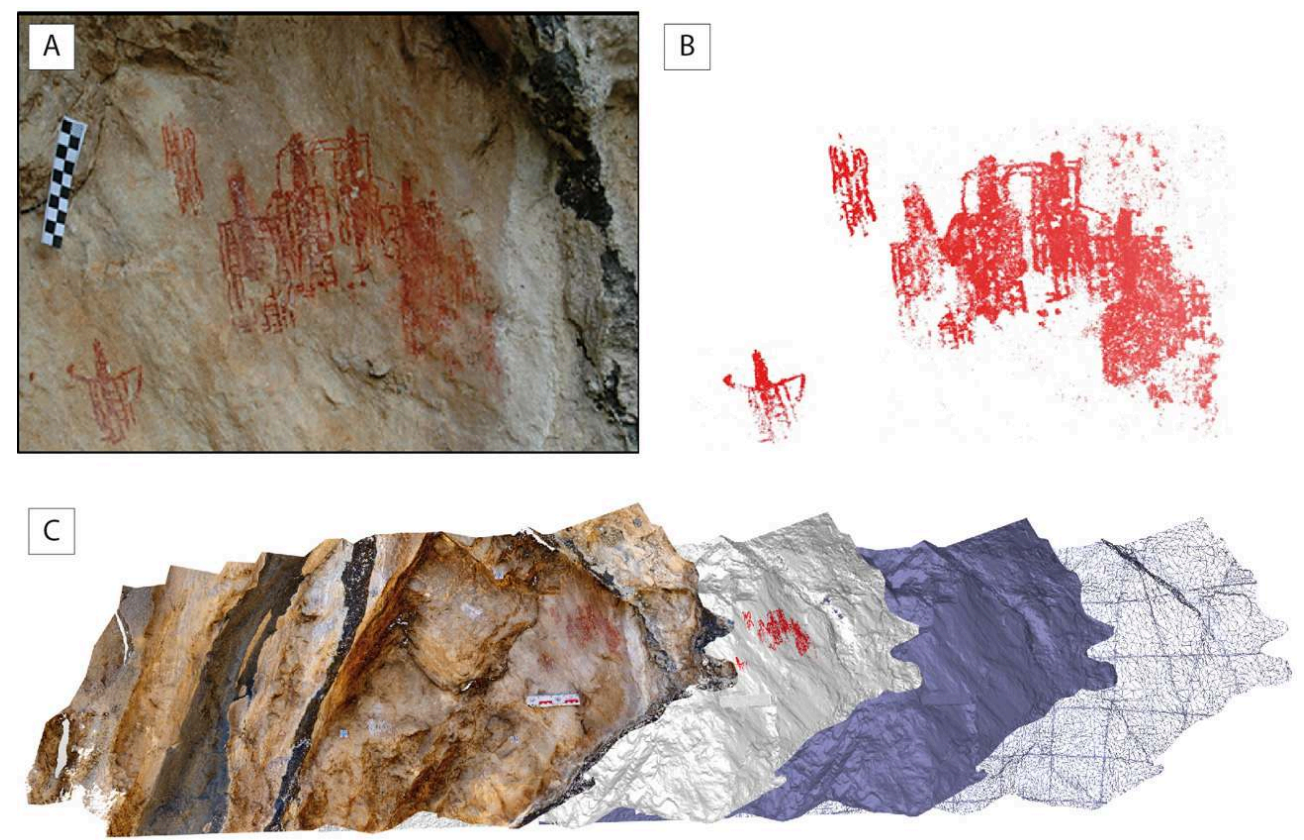

A. Photographie. B. Relevé digital. C. De gauche à droite : modèle photogramétrique texturé du panneau des peintures, caractérisation graphique (peintures et relevé 3D), mesh du modèle 3D, structure en fil de fer 3D.

G. Palmerini/PNM

\section{Perspectives}

Notre double enquête géoarchéologique veut reconstituer les processus d'installations humaines dans l'environnement karstique de moyenne et de haute montagne du massif de la Maiella et de ses environs. La mission de terrain 2021 a permis d'explorer 12 sites ornés de la Maiella et d'observer une séquence stratigraphique alluviale du Pléistocène en grotte.

Seul 30\% des sites d'art rupestre connus ont été analysés jusque-là. Tous souffrent de l'érosion naturelle, de façon plus ou moins accentuée: desquamation, lessivage, gélifraction, formations d'algues, lichens, champignons. Les peintures de cinq sites sont également menacées par l'activité humaine (parois d'escalade, dégradations intentionnelles). Dans six sites, l'altération a fait disparaître les peintures, pourtant signalées en 2007 par T. Mattioli ${ }^{40}$. Ces données sont transmises au Parc national de la Maiella afin d'évaluer les moyens à mettre en œuvre pour la conservation des peintures (signalétique, analyses des contaminations biologiques).

Les premiers résultats des analyses pXRF obtenus au cours de la mission de 2021 permettent d'envisager plusieurs perspectives. Concernant les pigments rouges, la présence de pigments de compositions différentes laisse supposer l'utilisation de plusieurs matériaux d'origine géologique distincte. Une approche de recherche de sources de matières premières pourrait donc être entreprise. Cette approche nécessite par ailleurs la quantification des concentrations en éléments discriminants présents dans les oxydes de fer tels que l'aluminium, le silicium, l'arsenic, etc. Concernant les pigments noirs, comme indiqué précédemment, la réalisation de datation directe par 
carbone 14 semble possible compte tenu de l'épaisseur relativement importante de matière sur certaines figures. La planification d'un échantillonnage des parois pourrait être envisagée dès 2022. Actuellement, ces peintures sont attribuées à une vaste période comprise entre la fin du Paléolithique et le Moyen Âge ${ }^{41}$. Du point de vue des sites du Paléolithique, la révision des données bibliographiques et l'analyse de la distribution des sites effectuée au prisme d'un SIG a permis d'identifier dans la Maiella orientale une zone à forte concentration de dépôts archéologiques en grottes datant du Paléolithique moyen et supérieur. Ces grottes ne semblaient pas accessibles aux groupes humains du Paléolithique inférieur, l'ouverture des canyons prenant place au mieux dans la deuxième partie du Pléistocène moyen. Les remplissages sédimentaires de certaines cavités (en particulier Grotta Scura) méritent une étude précise, afin d'apporter une meilleure contextualisation chronologique et environnementale aux vestiges archéologiques. Afin de découvrir de nouvelles séquences stratigraphiques du Pléistocène, des prospections systématiques dans les grottes de la Maiella orientale sont souhaitées.

\section{BIBLIOGRAPHIE}

Agostini et al. 1983

Silvano Agostini, Adelaide Rossi, Ezio Burri, Giovanni Masciarelli, « Una ricerca multidisciplinare: Grotta Scura (Bolognano) », Notiziario Speleo Club Chieti, 1983, p. 48-52.

Agostini, Rossi M.A. 1988

Silvano Agostini, Adelaide Rossi, « I sedimenti carsici e l'evoluzione geologica della Valle dell'Orta (Abruzzo Italia) », Notiziario Circolo Speleologico Romano n.s. 3, 1988, p. 135-162.

Agostini, Rossi M.A. 1993

Silvano Agostini, Adelaide Rossi, « Il carsismo della Maiella (Abruzzo) », in Atti XVI Congresso

Nazionale di Speleoleologia, Le grotte d'Italia (4) XVI, 1992-1993, p. 31-40.

ALHAIQUE, BIETTI 2008

Francesca Alhaique, Amilcare Bietti, « Il livello di conoscenza sulle strategie di sussistenza, Il Tardiglaciale », in Margherita Mussi (dir.), Italia - Lavori in corso, British Archaeological Reports International Series 1859, Oxford, Archaeopress, 2008, p. 21-33.

ANATI 1964

Emmanuel Anati, Civiltà preistorica in Valcamonica, Milano, Il Saggiatore ed., 1964.

BOSCHIAN 1995

Giovanni Boschian, «The "San Bartolomeo" shelter: a flint exploitation site in Central Italy ", Archaeologia Polona, 33, 1995, p. 31-40.

BREUIL 1933a

Henri Breuil, Les peintures rupestres schématiques de la Péninsule ibérique, I : au nord du Tage, Lagny, Imprimerie de Lagny, 1933. 
BREUIL 1933b

Henri Breuil, Les peinture rupestres schématiques de la Péninsule ibérique, II : Bassin du Guadiana, Lagny, Imprimerie de Lagny, 1933.

BREUIL 1933C

Henri Breuil, Les peintures rupestres schématiques de la Péninsule ibérique, III : Sierra Morena ", vol. III, Lagny, Imprimerie de Lagny,1933.

CONTI 1940

Carlo Conti, "Scoperta della più antica fase delle incisioni rupestri di Monte Bego », Bullettino di paletnologia italiana, 59, anno IV, 1940, p. 3-25.

CREMONESI et al. 1964

Giuliano Cremonesi, Cesare Occhiolini, Bertolucci Paolo, « Ricerche preistoriche in Abruzzo. Anno 1964 », Atti della Società Toscana di Scienze naturali, ser. A, 72, 1964, p. 508-514.

DEMANGEOT 1963

Jean Demangeot, « Karst et volcanisme en Italie centrale », Revue de géographie alpine, 51/2, 1963, p. 361-367.

DEMANGEOT, RADMILLI 1953

Jean Demangeot, Antonio Mario Radmilli, « Le gisement quaternaire de la Valle Giumentina (Abruzzes adriatiques), Stratigraphie et Palethnologie », Contributi di Scienze Geologiche, suppl. à La Ricerca Scientifica 3, 1953, p. 11-123.

DUMOULIN et al. 2020

Jean-Pascal Dumoulin, Matthieu Lebon, Ingrid Caffy, Guilhem Mauran, Alma Nankela, David Pleurdeau, Emmanuelle Delqué-Količ, Christophe Moreau, Marion Perron, Marc Sieudat, «Calcium oxalate radiocarbon dating: preliminary tests to date rock art of the decorated open-air caves, Erongo mountains, Namibia ", Radiocarbon, 62, 2020, p. 1551-15621-12. DOI: $10.1017 /$ RDC.2020.81

FELLER 1998

Laurent Feller, Les Abruzzes médiévales. Territoire, économie et société en Italie centrale $d u I^{e} a u$ XII siècle, BEFAR 300, Rome, École française de Rome,1998.

\section{FOSSATI 1991}

Alberto Fossati, «L'età del ferro nelle incisioni rupestri della Valcamonica », in Immagini di un'aristocrazia dell'età del Ferro, Catalogo della Mostra a Castello Sforzesco, aprile 1991-marzo 1992, Milano, 1991, p. 17-71.

GRAZIOSI 1973

Paolo Graziosi, L'arte preistorica in Italia, Firenze, 1973.

GRIFONI CREMONESI, TOSATTI 2017

Renata Grifoni Cremonesi, Anna Maria Tosatti, L'arte rupestre dell'età dei metalli nella penisola italiana: localizzazione dei siti in rapporto al territorio, simbologie e possibilità interpretative, Oxford, Archaeopress, 2017.

HAMEAU 1989

Philippe Hameau, Les peintures postglaciaires en Provence : inventaire étude chronologique, statistique et iconographique, Document d'archéologie française, 22, Paris, Editions de la Maison des sciences de l'Homme, 1989. 
HAMEAU 2002

Philippe Hameau, Passage, transformation et art schématiques : l'exemple des peintures néolithique du sud de la France, British Archaeological Reports, 1044, Oxford, Archaeopress, 2002.

LIBERATOSCIOLI et al. 2018

Elena Liberatoscioli, Giancarlo Boscaino, Silvano Agostini, Adele Garzarella, Etta Patacca

Scandone, « The Majella National Park: An Aspiring UNESCO Geopark », Geosciences 2018, 8 (7), 256, 2018, p. 1-21.

MAGGIORI, MUCCIANTE 2000-2001

Marcello Maggiori, Alessandro Mucciante, « Una stratigrafia in grotta con industria del paleolitico superiore nei pressi di Pretoro (Pescara) ", Atti della Società toscana di Scienze naturali, ser. A, 2000-2001, p. 33-39.

MATTIOLI 2007

Tommaso Mattioli, L'arte rupestre in Italia centrale: Umbria, Lazio, Abruzzo, Quaderni di Protostoria, 4, Perugia, Ali\&no, 2007.

MAzzA et al. 2005

Paul Mazza, Marco Rustioni, Silvano Agostini, Adelaide Rossi, « An unexpected Late Pleistocene macaque remain from Grotta degli Orsi Volanti (Rapino, Chieti, Central Italy) », Geobios, 38, 2005, p. 211-217.

MORETTI 1968

Mario Moretti, Architettura medioevale in Abruzzo: dal vi secolo al XVI secolo, L'Aquila, Camera di Commercio degli Abruzzi e Molise, 1968.

Nicoud et al. 2013

Elisa Nicoud, Marina Pagli, Daniele Aureli, Silvano Agostini, Giovanni Boschian, Christine Chaussé, Jean-Philippe Degeai, Fabio Fusco, Catherine Kuzucuoglu, Paolo Mazza, Valentina Villa, « Valle Giumentina (Abruzzes, Italie) », Chronique des activités archéologiques de l'EFR, 2013, en ligne, http://cefr.revues.org/902

Nicoud et al. 2016

Elisa Nicoud, Daniele Aureli, Marina Pagli, Valentina Villa, Christine Chaussé, Silvano Agostini, Jean-Jacques Bahain, Giovanni Boschian, Jean-Philippe Degeai, Fabio Fusco, Biaggio Giaccio, Marion Hernandez, Catherine Kuzucuoglu, Christelle Lahaye, Cristina Lemorini, Nicole LimondinLozouet, Paolo Mazza, Norbert Mercier, Sébastien Nomade, Alison Pereira, Vincent Robert, Maria Adelaide Rossi, Clément Virmoux, Andrea Zupancich, « Preliminary data from Valle Giumentina Pleistocene site (Abruzzo, Central Italy): A new approach to a Clactonian and Acheulian sequence », Quaternary International 409B, 2016, p. 182-194.

Nicoud et al. 2020

Elisa Nicoud, Valentina Villa, Gourguen Davtian, Juliette Guibert-Cardin, « Valle Giumentina (Abruzzes), Huitième mission : étude des comportements techno-économiques au Pléistocène moyen », Chronique des activités archéologiques de l'EFR, 2020, en ligne, http:// journals.openedition.org/cefr/4206

PALMERINI 2017

Guido Palmerini, « Handprints in rock art, note preliminari dall'Abruzzo », Bollettino del Centro Camuno di Studi Preistorici, 43, 2017, p. 1-16.

PALMERINI et al. 2021

Guido Palmerini, Lucile Beck, Luciano Di Martino, Xavier Gallet, Matthieu Lebon, Aurelio Manzi, Elisa Nicoud, Antonio Mariano Spera, Valentina Villa, « \#MaiellaRockArtProject: Nuove ricerche 
sull'arte rupestre dell'Appennino abruzzese ", in Proceedings of the XXVIII Valcamonica Symposium, Centro Camuno di Studi Preistorici, 2021, p. 35-40.

PRIULI 1991

Ausilio Priuli, La cultura figurativa preistorica e di tradizione in Italia, Pesaro, Giotto Printer ed., 1991.

RADMILLI 1977

Antonio Mario Radmilli, Storia dell'Abruzzo dalle origini all'età del bronzo, Pisa, 1977, 464 p.

RADMILLI 1993

Antonio Mario Radmilli, L'Abruzzo dal Paleolitico inferiore alle genti Italiche. Ricerche di paletnologia in territorio abruzzese a carattere scientifico-divulgativo, I tascabili d'Abruzzo, 58, Polla, 1993, 155 p.

RADMILLI 1997

Antonio Mario Radmilli, La vita in Abruzzo ventimila anni fa. Il Paleolitico Superiore, Pisa, ETS ed., $1997,275 \mathrm{p}$.

RADMILLI, MALLEGNI, FORNACIARI 1978

Antonio Mario Radmilli, Francesco Mallegni, Gino Fornaciari, « Recenti scavi nella Grotta dei Piccioni di Bolognano (Pescaro) e riesame dei resti scheletrici umani provenienti dai circoli », Atti della Società Toscana di Scienze Naturali, 85, 1978, p. 175-198.

RUGGERI, WHALLON 2010

D. Ruggeri, Robert Whallon, «Sant'Angelo Cave - a Gravettian huntingand raw material procurement site in theMaiella Mountains, Abruzzo (Italy) », Human Evolution, 25, 1-2, 2010, p. 67-82.

SACCÀ 2016

Daniela Saccà, « I resti faunistici del sito musteriano di Grotta Rutina », in Atti del $7^{\circ}$ Convegno Nazionale di Archeozoologia, Annali dell'Università degli Studi di Ferrara Museologia Scientifica e Naturalistica, 12/1, 2016, p. 27-34.

SOLÉ et al. 2007

Vincente Armando Solé, Emmanuel Papillon, Marine Cotte, Philippe Walter, Jean Susini, «A multiplatform code for the analysis of energy-dispersive X-ray fluorescence spectra ", Spectrochimica Acta Part B: Atomic Spectroscopy, 62/1, 2007, p. 63-68.

TOZZI 1982

Carlo Tozzi, « Industria del Paleolitico Inferiore lungo il fiume Foro e alla Selvotta (Chieti), dans in Atti della XXIII riunione scientifica dell'Istituto italiano di Preistoria e Protostoria, Firenze, 1982, p. 511-529.

TOZZI 2003

Carlo Tozzi, « Il Paleolitico dell'Abruzzo », Atti della XXXVI riunione scientifica dell'Istituto italiano di Preistoria e Protostoria, Firenze, 2003, p. 7-27.

VILLA et al. 2016

Valentina Villa, Alison Pereira, Christine Chaussé, Sébastien Nomade, Fabio Fusco, Nicole Limondin-Lozouet, Jean-Philippe Degeai, Vincent Robert, Catherine Kuzucuoglu, Giovanni Boschian, Daniele Aureli, Marina Pagli, Elisa Nicoud, « A MIS 15-MIS 12 record of environmental changes and Lower Palaeolithic occupation from Valle Giumentina, central Italy », Quaternary Science Reviews 151, 2016, p. 160-184.

VILLA et al. 2021a

Valentina Villa, Elisa Nicoud, Fabio Fusco, «Valle Giumentina (Abruzzes), neuvième mission : 
étude paléoenvironnementale des occupations humaines du Pléistocène moyen ", Bulletin archéologique des Ecole françaises à l'étranger, 2021, en ligne, https://doi.org/10.4000/baefe.1247 VILLA et al. 2021b

Valentina Villa, Elisa Nicoud, Fabio Fusco, Alison Pereira, Sébastien Nomade, Juliette GuibertCardin, Christine Chaussé, Gourguen Davtian, « Human settlements during a cold and dry event of MIS $13 \mathrm{~b}$ in Central Italy (ABF-LAC Levels of valle Giumentina) », in Actes des $41^{\text {ème }}$ rencontres internationales d'Archéologie et d'Histoire de Nice, Biodiversité, environnements et sociétés depuis la Préhistoire, Nice, APDCA-CEPAM, 2021, p. 151-168.

\section{NOTES}

1. e. g. NICOUD et al. 2013, 2016, 2020.

2. Liberatoscioli et al. 2018. 95 géosites «d'intérêt mondial » ont été identifiés dans le PNM à l'occasion de sa candidature au label Unesco Geopark.

3. e. g. VILLA et al. 2016, 2021a, $2021 \mathrm{~b}$.

4. https://adriaticaves.adrioninterreg.eu.

5. Projet de G. Palmerini, Université Côte d'Azur CEPAM, encadré par R.M. Dessì, en collaboration avec le PNM ; PALMERINI et al. 2021.

6. DEMANGEOT 1963.

7. AGOSTINI et al. 1983, AGOSTINI, ROSSI 1993.

8. AgOSTINI, ROSSI 1988.

9. PALMERINI et al. 2021.

10. DEMANGEOT, RADMILLI 1953, RADMILLI 1993.

11. Tozzi 2003.

12. ALHAIQUE, BIETTI 2008, RUGGERI, WHALLON 2010.

13. RADMILLI 1997.

14. TozzI 1982.

15. MAZZA et al. 2005.

16. ALHAIQUE, BIETTI 2008, RUGGERI, WHALLON 2010.

17. MAGGIORI, MUCCIANTE 2000-2001.

18. SACCÀ 2016.

19. RADMILLI 1993.

20. BOSCHIAN 1995.

21. RADMILLI, MALLEGNI, FORNACIARI 1978.

22. RADMILLI 1993.

23. RADMILLI 1993.

24. FELLER 1998.

25. MORETTI 1968.

26. GRAZIOSI 1973, PRIULI 1991.

27. ANATI 1964, FOSSATI 1991. 
28. CREMONESI et al. 1964.

29. GRIFONI CREMONESI, TOSATTI 2017.

30. MATTIOLI 2007.

31. CONTI 1940 ; HAMEAU 1989, 2002 ; BREUIL 1933a-C.

32. MATTIOLI 2007.

33. AGOSTINI et al. 1983.

34. PALMERINI 2017.

35. MATTIOLI 2007, p. 63-64.

36. RADMILLI 1977, p. 422-423.

37. MATTIOLI 2007, p. 63-64 ; PALMERINI et al. 2021, p. 37.

38. SOLÉ et al. 2007.

39. Dumoulin et al. 2020.

40. MATTIOLI 2007.

41. MATTIOLI 2007.

\section{INDEX}

\section{Année de l'opération : 2021}

sujets https://ark.frantiq.fr/ark:/26678/pcrtmkRNd3ikl4, https://ark.frantiq.fr/ark:/26678/ pcrtM6WKp5XFlj, https://ark.frantiq.fr/ark:/26678/pcrtI7E5QGYFhb, https://ark.frantiq.fr/ark:/ 26678/pcrtpSis9hZLUb, https://ark.frantiq.fr/ark:/26678/pcrtkeLpJpVN4t, https:// ark.frantiq.fr/ark:/26678/pcrtET0l280TUS, https://ark.frantiq.fr/ark:/26678/pcrtGQAvQ8BYiP, https://ark.frantiq.fr/ark:/26678/pcrtGzQPnntJa1 chronologie https://ark.frantiq.fr/ark:/26678/pcrtFS9v4PxyYL

Thèmes : EFR

\section{AUTEURS}

\section{ELISA NICOUD}

Université Côte d'Azur, CNRS UMR 7264 CEPAM

\section{GUIDO PALMERINI}

Université Côte d'Azur, CNRS UMR 7264 CEPAM

\section{VALENTINA VILLA}

Université Côte d'Azur, CNRS UMR 7264 CEPAM

\section{SILVANO AGOSTINI}

Université Côte d'Azur, CNRS UMR 7264 CEPAM 


\section{LUCILE BECK}

Université Côte d'Azur, CNRS UMR 7264 CEPAM

ROSA MARIA DESSì

Université Côte d'Azur, CNRS UMR 7264 CEPAM

XAVIER GALLET

Università G. D’Annunzio Chieti-Pescara, CAAM

\section{MATTHIEU LEBON}

LMC14, LSCE/IPSL, CEA-CNRS-UVSQ, Université Paris-Saclay

DIRECTEURFOUILLES_DESCRIPTION

ELISA NICOUD

Université Côte d'Azur, CNRS UMR 7264 CEPAM

GUIDO PALMERINI

Université Côte d'Azur, CNRS UMR 7264 CEPAM

VALENTINA VILLA

Université Côte d'Azur, CNRS UMR 7264 CEPAM 